\title{
Efeitos agudos de diferentes intensidades de exercício sobre a ingestão alimentar pós-exercício
}

CDD. 20.ed. 796.027

796.071

\author{
Tatiana Acioli LINS* \\ Pedro Rogério da Silva NEVES* \\ Thiago Ricardo dos Santos TENÓRIO** \\ Aline Daniela CRUZ* \\ Carla Caroliny de Almeida SANTANA** \\ Wagner Luiz do PRADO***
}

\begin{abstract}
Resumo
0 objetivo do presente estudo foi verificar os efeitos agudos de diferentes intensidades de exercício aeróbio ( 40 e $80 \%$ do $\mathrm{VO}_{2 \text { pico }}$ ) sobre a ingestão alimentar pós-exercício. Participaram do estudo 18 adultos jovens, eutróficos $\left(22,20 \pm 1,72 \mathrm{~kg} / \mathrm{m}^{2}\right)$ e fisicamente ativos. Todos os sujeitos foram submetidos aleatoriamente a três condições experimentais: controle (sem exercício); EBI, exercício de baixa intensidade (40\% do $\left.\mathrm{VO}_{2 \text { pico }}\right)$ e EAl, exercício de alta intensidade $\left(80 \%\right.$ do $\mathrm{VO}_{2 \text { pico }}$ ). As sessões de exercicio foram isocalóricas (350 kcal). Após 120 minutos de recuperação passiva, os voluntários tinham livre acesso a um "buffet" variado de alimentos, a ingestão alimentar foi determinada através da pesagem dos alimentos ingeridos. Os dados alimentares obtidos foram então tabulados e analisados por meio do "software" Nutwin 6.0 (UNIFESP, 2002), para estimativa do consumo energético total (kcal) e ingestão dos macronutrientes (carboidratos, proteínas e lipídeos) em gramas. Os resultados não demonstram nenhuma diferença na ingestão alimentar entre as condições experimentais analisadas. Dessa forma, podemos concluir que a ingestão alimentar pós-exercício não se mostrou dependente da intensidade do esforço em curto prazo em indivíduos adultos jovens fisicamente ativos.
\end{abstract}

Unitermos: Exercício aeróbio; Comportamento alimentar; Metabolismo energético.

\section{Introdução}

Há mais de 50 anos, a relação entre o gasto energético (GE) promovido pelo exercício e a ingestão alimentar (IA) tem sido foco de investigação de pesquisadores interessados em compreender melhor o balanço energético (BE) (Blundell, Stubbs, Hughes, Whybrow \& KING, 2003). Atualmente sabe-se que após a realização de esforços físicos, ocorrem ajustes compensatórios no organismo que visam controlar, entre outros aspectos, o GE, a IA, a sensação de fome/saciedade e o apetite (Broom, Batterham, King \& Stensel, 2009).

Recentemente, Lins, Neves, Costa e Prado (2010) demonstram que o exercício físico é capaz de modular o comportamento alimentar, mesmo com moderado gasto energético e em estados de balanço energético equilibrado (neutro), tais alterações no comportamento alimentar ocorrem através da indução de um estado transitório de supressão da fome e do apetite (Bilski, Teległów, Zahradnik-BilsKa, Dembinski \& Warzecha, 2009; Broom, Stensel, Bishop, Burns \& Miashyta, 2007; Broom et al., 2009; Cheng, Burshnell, Cannon \& Kern, 2009).

Entretanto, alguns estudos verificaram haver um efeito compensatório (aumento) na sensação de fome e apetite (ERdmann, Tahbaz, Lippl, Wagenpfeil \& Schusdziarra, 2007; Martins, Morgan, Bloom \& Robertson, 2007; Whybrow, Hughes, Ritz, Johnstone, Horgan, King, Blundell \& Stubbs, 2008) enquanto que outros não observaram nenhum efeito do exercício aeróbio sobre a sensação de fome/ apetite (Imbeault, Saint-PirRe, Almeras \& Tremblay, 1997; King \& Blundell, 1995; Westerp-Platenga, VerWEgen, IJEDEMA, WiJCKMANS \& SaRIS, 1997). 
A sensação de fome, apetite e saciedade são preditores confiáveis da ingestão alimentar pós-exercício (Drapeau, King, Hetherington, Doucet, BlunDELl \& Tremblay, 2007), porém medidas diretas de IA devem ser utilizadas para se quantificar os reais efeitos do exercício no comportamento alimentar. Utilizando-se de medidas diretas de IA, algumas pesquisas não verificaram efeitos do exercício sobre a IA (Hagobian, Sharoff \& Braun, 2008; Imbeault et al., 2005). Entretanto, pesquisas sugerem que o exercício físico agudo é capaz de reduzir a ingestão de alimentos, através de um fenômeno denominado "anorexia induzida pelo exercício” (KING, BuRley \& BLUNDELL, 1994; King, Lluch, Stubbs \& Blundell, 1997).

Postula-se que as influências do esforço físico sobre o comportamento alimentar são advindas do gasto energético promovido pela atividade (KING et al., 1997), porém, evidências demonstram que a intensidade do exercício desempenha um papel fundamental no controle do comportamento alimentar, ou seja, quanto maior a intensidade do exercício, menor a ingestão alimentar relativa (WESTERTERP-PLANTENGA et al., 1997), mais uma vez, tal hipótese não é consenso na literatura atual, visto que autores encontraram resultados contrários (GEORGE \& MoRgansteIn, 2003; Pomerlau, Imbeault, Parker \& Doucet, 2004).

Até o presente momento são escassos e inconclusivos os estudos que objetivaram verificar os efeitos de diferentes intensidades de exercício aeróbio sobre a ingestão alimentar pós-exercício, principalmente isolando-se o efeito clássico da indução de um estado de balanço energético negativo, advindo do gasto energético. Sendo assim, o objetivo do presente estudo foi verificar os efeitos agudos de diferentes intensidades de exercício aeróbio (40 e 80\% do $\mathrm{VO}_{\text {2pico }}$ ) sobre a ingestão alimentar pós-exercício.

\section{Métodos}

Este estudo caracteriza-se como experimental, "crossover" (cruzado) e aleatorizado.
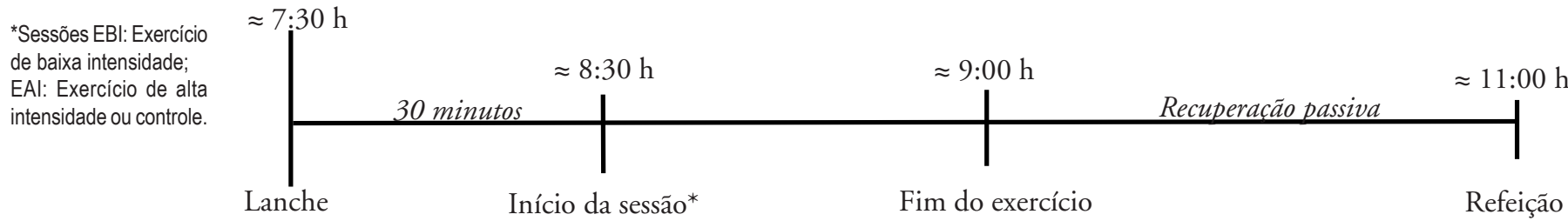

FIGURA 1 - Desenho experimental do estudo.

\begin{abstract}
Amostra
Foram selecionados 20 indivíduos adultos jovens do gênero masculino, estudantes universitários, que se voluntariaram a participar da pesquisa, após leitura de cartazes/convite distribuídos pelo campus da universidade. Foram estabelecidos como critérios de inclusão: idade entre 18 e 25 anos, prática regular de exercício físico ( $\geq$ seis meses, pelo menos três vezes por semana) (IPAQ) e eutróficos (18,5 < IMC < 24,9 $\mathrm{kg} / \mathrm{m}^{2}$ ); e critérios de exclusão: consumo crônico de álcool, tabaco, fármacos ou uso de suplementos alimentares; ser portador de doença genética, endócrina ou metabólica; e apresentar variação na massa corporal (>2 kg) nos últimos seis meses.
\end{abstract}

Os voluntários assinaram termo de consentimento livre e esclarecido, e o estudo foi aprovado pelo Comitê de Ética da Universidade de Pernambuco (\#154/08). Todos os procedimentos foram realizados no laboratório de avaliação da performance humana (LAPH) da Escola Superior de Educação Física (ESEF) da Universidade de Pernambuco (UPE).

Dos 20 voluntários que iniciaram o estudo, cinco não completaram todas as sessōes experimentais (dois apresentaram problemas de saúde, dois desistiram e um não conseguiu cumprir o protocolo na sessão de alta intensidade), dessa forma, a TABELA 1 apresenta as características antropométricas dos 15 voluntários que completaram as sessões experimentais. 
TABELA 1 - Características antropométricas, composição corporal e consumo de oxigênio dosjovens submetidos a diferentes intensidades de exercício aeróbio.

\begin{tabular}{|c|c|}
\hline Variáveis & Média + D.P \\
\hline Idade (anos) & $21 \pm 2$ \\
\hline Massa corporal (kg) & $66,50 \pm 7,10$ \\
\hline Estatura $(\mathrm{cm})$ & $174,00 \pm 7,00$ \\
\hline $\operatorname{IMC}\left(\mathrm{kg} / \mathrm{m}^{2}\right)$ & $22,20 \pm 1,72$ \\
\hline$\%$ Gordura & $14,50 \pm 3,04$ \\
\hline $\mathrm{VO}_{2 \text { pico }}(\mathrm{L} / \mathrm{min})$ & $3,31 \pm 0,40$ \\
\hline $\mathrm{VO}_{2 \text { pico }}(\mathrm{ml} / \mathrm{kg} / \mathrm{min})$ & $50,00 \pm 5,30$ \\
\hline
\end{tabular}

IMC= índice de massa corporal;

$\mathrm{VO}_{2 \text { pico }}=$ consumo pico de oxigênio.

\section{Procedimentos}

\section{Delineamento experimental}

Os sujeitos realizaram quatro visitas ao laboratório, durante a primeira visita foi mensurada a estatura, massa corporal, composição corporal e consumo de oxigênio $\left(\mathrm{VO}_{2}\right)$. As intensidades de esforço foram determinadas a partir do consumo pico de oxigênio $\left(\mathrm{VO}_{\text {2pico }}\right)$ obtido em teste ergoespirométrico máximo em esteira rolante. Todas as sessões experimentais foram conduzidas no mesmo período do dia (manhã), para evitar quaisquer influências circadianas, em ambiente com temperatura constante $\left(24 \pm 1^{\circ} \mathrm{C}\right)$. Os sujeitos foram orientados a não realizar nenhum tipo de exercício físico $48 \mathrm{~h}$ antes de cada sessão experimental.

Da segunda até a quarta visita os sujeitos foram submetidos aleatoriamente (ramdomizer.org), e com intervalo de sete dias, as sessões experimentais: 1) controle: repouso por 30 minutos (sentados); 2) Exercício de baixa intensidade (EBI): exercício físico em esteira rolante em intensidade de esforço correspondente a $40 \% \mathrm{VO}_{2 \text { pico }}$; 3) Exercício de alta intensidade (EAI): exercício físico em esteira rolante em intensidade de esforço correspondente a $80 \% \mathrm{VO}_{2 \text { pico }}$.

Para a realização das sessões experimentais, os sujeitos chegaram ao laboratório às 7:30h, após jejum noturno de $12 \mathrm{~h}$, momento em que consumiram um lanche padrão [um pão francês ( $50 \mathrm{~g})$, um fatia de queijo prato $(30 \mathrm{~g})$ e um copo de suco frutas $(200 \mathrm{ml})]$ com densidade energética de $350 \mathrm{kcal}$ (Carboidrato: 61,7\%; Proteína: 13,44\% e Lipídeos: 24,86\%), 30 minutos após a ingestão do lanche $(\approx$ às 8:00h) iniciava-se a sessão experimental.

Todas as sessões de exercício físico foram isocalóricas, com gasto calórico de $\approx 350 \mathrm{kcal}$. Após o término da sessão de exercício (ou 30 minutos para condição controle), os sujeitos permaneceram sentados (recuperação passiva) por
120 minutos, após este período a ingestão alimentar foi determinada pela pesagem e análise da refeição que foi ofertada "ad libitum". Durante todo o período experimental (exercício + recuperação) foi realizada análise direta de gases para determinação do gasto calórico através de calorimetria indireta.

\section{Medidas antropométricas e de composição corporal}

A massa corporal foi obtida através de balança (Fillizola, Brasil) com escala de precisão $0,1 \mathrm{~kg}$, a estatura foi mensurada através de estadiômetro de madeira montado, com precisão de $0,1 \mathrm{~mm}$, seguindo a metodologia proposta por JACKSON e POLLOCK (1984). Posteriormente foi calculado o índice de massa corpórea (IMC). A composição corporal foi predita pelo método de impedância bioelétrica (Biodynamics (USA) modelo A-310 "body composition analyzing”), observando-se os critérios descritos pelo manual do equipamento e demais procedimentos previamente estabelecidos na literatura (LUCKASKI, BolonchuK, Hall \& Siders, 1986).

\section{Consumo de Oxigênio (VO2)}

O consumo máximo de oxigênio (VO2) foi determinado através de teste incremental em esteira rolante (Inbrasport Super ATL, Brasil), utilizando-se o protocolo proposto por MCCONNEL (1988). A inclinação da esteira foi mantida constante em $1,0 \%$, com velocidade inicial de $5,0 \mathrm{~km} / \mathrm{h}$ (aquecimento por três minutos), seguidos de incrementos de $1,0 \mathrm{~km} / \mathrm{h}$ a cada minuto.

Os critérios estabelecidos para interrupção do teste foram: Fadiga voluntária, Quociente Respiratório acima de 1,15 e Escala de Borg acima de 18 . O maior valor de $\mathrm{VO}_{2}$ encontrado antes da interrupção do teste foi adotado como sendo o consumo pico de oxigênio $\left(\mathrm{VO}_{2 \text { pico }}\right)$. 
$\mathrm{O} \mathrm{VO}_{2}$ e a produção de dióxido de carbono $\left(\mathrm{VCO}_{2}\right)$ foram obtidos em um analisador metabólico de circuito aberto (Córtex Biophysik Metalyzer IIB, Alemanha) com leitura a cada 15 segundos, com utilização de máscara (Hans Rudolph Linc, EUA).

O sistema foi calibrado para volume (seringa de calibração de volume- 3L Cardioequipo, Brasil), pressão atmosférica (Barômetro- Barigo, Alemanha) e mistura de gases (White Martins- concentração- $\mathrm{O}_{2}=$ 12,2 e $\left.\mathrm{CO}_{2}=4,8\right)$ antes de cada teste seguindo todas as recomendaçôes do fabricante. $\mathrm{O} \mathrm{VO}_{2}$ e a $\mathrm{VCO}_{2}$ foram avaliados durante todo o período experimental (exercício e controle +120 minutos de recuperação).

\section{Gasto energético / Taxa Metabólica}

Durante todo o procedimento experimental os voluntários tiveram o $\mathrm{VO}_{2}$ e $\mathrm{VCO}_{2}$ constantemente monitorados (Córtex Biophysik Metalyzer IIB, Alemanha) para determinação do gasto energético através de calorimetria indireta mensurada no "steady state". O somatório do consumo total de $\mathrm{O}_{2}$ foi utilizado para calcular o dispêndio energético total, utilizando o valor calorífico de 4,825 kcal de energia para cada litro de $\mathrm{O}_{2}$ consumido (KRAUSE \& MAHAN, 1991).

\section{Ingestão Alimentar pós-exercício}

O preparo dos alimentos ofertados foi previamente analisado, a fim de minimizar erros

\section{Resultados}

Uma vez que o gasto calórico das sessões foi estabelecido em 350 kcal, não houve diferença no gasto energético nas diferentes intensidades de exercício (TABELA 2), porém, o EAI induziu a um maior gasto calórico pós-exercício que o $\mathrm{EBI}(\mathrm{EBI}=60,19 \pm 5,54$; $\mathrm{EAI}=$ $67,95 \pm 5,14 ; \mathrm{p}<0,05)$, houve diferença significativa na duração das sessões $(\mathrm{EBI}=59,68 \pm$ 5,02 min; EAI $=29,43 \pm 2,52 \mathrm{~min}, \mathrm{p}<0,004)$, e na velocidade $(\mathrm{EBI}=$ $5,6 \pm 0,34 \mathrm{~km} / \mathrm{h} ; \mathrm{EAI}=11,28 \pm 0,77 \mathrm{~km} / \mathrm{h}, \mathrm{p}<0,001)$.

Observando a contribuição absoluta dos macronutrientes para o fornecimento de energia, os dados revelam que houve uma maior mobilização na estimativa dos macronutrientes. Após 120 minutos de recuperação passiva, os voluntários, individualmente, tiveram livre acesso a um "buffet" variado de alimentos, onde lhes era permitido comer a vontade. Cada alimento colocado no prato era pesado em uma balança com precisão de $20 \mathrm{~g}$ e capacidade máxima de 2,2 kg (Kwonnie, Japão), sem que os voluntários tivessem acesso aos valores obtidos. A repetição da refeição era permitida, respeitando-se os mesmos procedimentos adotados na primeira pesagem. As sobras no prato também eram pesadas e subtraídas do valor total.

Os dados foram tabulados e analisados utilizando-se o "software" Nutwin 6.0 (ANÇĀo, CUPPARI, Draibe \& Sigulem, 2002), para determinação do valor energético total (VET) (kcal) e ingestão específica de carboidratos $(\mathrm{CHO})$, proteínas (AA) e lipídeos (LIP), em gramas.

\section{Análise estatística}

Todos os procedimentos estatísticos foram realizados através do "Software Statistica" versão 6.0 (STAT SOFT). Foi realizado o teste de normalidade de Shapiro Wilk e homogeneidade de Levene. ANOVA One-Way para medidas repetidas foi utilizada para analisar os efeitos das sessões experimentais (EBI, EAI e Controle) sobre a ingestão alimentar. Os valores são apresentados em média \pm desvio padrão. $O$ nível de significância foi fixado com $\mathrm{p} \leq 0,05$.

de carboidratos e de lipídeos nas sessões de exercício em relação à sessão controle $(\mathrm{p}<0,002)$, e um maior gasto de carboidratos durante o EAI quando comprado ao EBI $(p<0,001)$, entretanto, houve uma maior oxidação lipídica em resposta ao EBI com relação ao EAI ( $\mathrm{p}<0,004)$ (TABELA 2).

A TABELA 3 apresenta os valores referentes à ingestão alimentar pós-exercício, observa-se que não foram encontradas diferenças significativas no VET (kcal), bem como na ingestão específica dos macronutrientes, entre todas as condiçōes experimentais (EBI, EAI e Controle). 
TABELA 2 - Efeitos de diferentes intensidades de exercício aeróbio sobre o gasto energético total e sobre a mobilização de diferentes substratos energéticos durante o exercício.

\begin{tabular}{lccc}
\hline & Controle $(\mathbf{n}=\mathbf{1 8})$ & EBI $(\mathbf{n}=\mathbf{1 6})$ & EAI $(\mathbf{n}=\mathbf{1 5})$ \\
\hline Tempo (minutos) & 120 & $59,68 \pm 5,02$ & $29,43 \pm 2,52^{\mathrm{ab}}$ \\
Gasto Energético (kcal) & $60,17 \pm 5,40$ & $339,35 \pm 20,16^{\mathrm{a}}$ & $341,75 \pm 17,61^{\mathrm{a}}$ \\
Gasto de carboidrato (kcal) & $26,69 \pm 6,90$ & $228,12 \pm 40,94^{\mathrm{a}}$ & $281,74 \pm 58,52^{\mathrm{ab}}$ \\
Gasto de lipídeos $(\mathrm{kcal})$ & $33,58 \pm 8,20$ & $111,22 \pm 40,77^{\mathrm{a}}$ & $60,00 \pm 55,77^{\mathrm{ab}}$ \\
\hline
\end{tabular}

${ }^{a}$ vs Controle; bvs EBI;

$\mathrm{EBI}=$ Exercício de Baixa Intensidade;

EAl = Exercício de Alta Intensidade; $p \leq 0,05$; ANOVA One-Way post hoc de Tuckey.

TABELA 3 - Efeitos de diferentes intensidades de exercício sobre a ingestão alimentar.

\begin{tabular}{lccc}
\hline & Controle $(\mathbf{n}=\mathbf{1 8})$ & EBI $(\mathbf{n}=\mathbf{1 6})$ & EAI $(\mathbf{n}=\mathbf{1 5})$ \\
\hline VET $(\mathrm{kcal})$ & $960,14 \pm 213,15$ & $938,57 \pm 239,24$ & $992,55 \pm 238,66$ \\
CHO (kcal) & $506,72 \pm 66,77$ & $489,52 \pm 135,77$ & $513,33 \pm 158,01$ \\
AA $(\mathrm{kcal})$ & $206,72 \pm 66,77$ & $203,45 \pm 80,09$ & $225,38 \pm 67,35$ \\
LIP $(\mathrm{kcal})$ & $246,82 \pm 106,50$ & $241,22 \pm 115,85$ & $245,44 \pm 99,37$ \\
\hline
\end{tabular}

VET = Valor energético total; $\mathrm{CHO}=$ carboidrato $\mathrm{AA}=$ proteína; LIP = lipídeos; $\mathrm{EBI}=$ Exercício de Baixa Intensidade; EAI $=$ Exercício de Alta Intensidade; $p \leq 0,05$; ANOVA One-Way post hoc de Tuckey.

\section{Discussão}

A proposta deste estudo foi verificar os efeitos agudos de diferentes intensidades de exercício aeróbio sobre a ingestão alimentar pós-exercício.

A regulação da ingestão alimentar pode ser dividida em duas vias: uma de longo prazo, responsável pela manutenção das reservas energéticas corporais (especialmente de gordura), e uma de curto prazo, que em última instância controla a fome, a saciedade, o apetite e a ingestão alimentar, em cada refeição (Hagobian, Sharoff \& Braun, 2008).

Estudos têm demonstrado que o exercício físico agudo, associado ou não a um estado de déficit energético, pode estimular a sensação de fome (Maraki et al., 2005; Pomerleau et al., 2004; Verger, Lanteaume \& Louis-Sylvestre, 1992, 1994), acompanhado de um aumento na ingestão alimentar (Pomerleau et al., 2004; Verger, Lanteaume \& Louis-Sylvestre, 1994), enquanto outros verificaram supressão da ingestão alimentar (Moore, Dodd, Welsman \& Armstrong, 2004; Westerp-Plantenga et al., 1997).

Tais divergências nos resultados apresentados podem ser atribuídas, principalmente as diferentes intensidades de exercício utilizadas, pois, acredita-se que, quanto maior a intensidade do esforço, maior a supressão da IA, e que tais alteraçôes não são tão pronunciadas após exercícios de baixa ou moderada intensidade (BLUNDELl et al., 2003).

No presente estudo, mesmo utilizando-se duas intensidades de exercício físico e controlando-se a ingestão prévia, não foi encontrado alteração na ingestão alimentar após duas horas da realização de exercício, resultado este compartilhado por outros estudos da literatura (Blundell \& KING, 1999; George \& Morganstein, 2003; Lluch, King \& Blundell, 1998; Westerterp-Plantenga et al., 1997), tal resultado pode ser parcialmente explicado, pelo baixo déficit induzido pelo exercício, vale ressaltar que no presente estudo, o gasto foi estabelecido em $350 \mathrm{kcal}$, e os voluntários recebiam uma refeição prévia, com densidade energética também de 350 kcal.

Entretanto, BLUNDELL et al. (2003) observaram que, mesmo quando grandes déficits de energia são alcançados (> $400 \mathrm{kcal}$ ), não resultam em aumento da ingestão alimentar em curtos períodos de tempo. Neste mesmo sentido, BroOm et al. (2009), destacam que exercício agudo não estimula o consumo de energia em curto prazo (um a dois dias após o exercício), sendo tais alteraçôes mais evidenciadas após longos períodos de treinamento, e não em resposta aguda a uma única sessão.

Vale ressaltar que diversos fatores cognitivos podem influenciar o comportamento alimentar pós-exercício, através da estimulação/inibição de vias hedônicas, relacionadas ao prazer de se alimentar (Blundell \& King, 1999), e no presente estudo esta variável não foi controlada.

Em nosso estudo, o EAI mesmo induzindo a um maior GE pós-exercício não influenciou a IA, o que se deve, provavelmente, ao condicionamento dos sujeitos, 
uma vez que, indivíduos sedentários tendem a compensar a IA agudamente, diferente de indivíduos fisicamente ativos que não apresentam esse comportamento (STUBBS, Hughes, Johnstone, Horgan, King \& Blundell, 2004; WhyBrow et al., 2008).

A utilização de substratos energéticos durante o exercício aeróbio também não influenciou o comportamento alimentar pós-exercício, uma vez que nossos achados, não revelaram diferenças entre a escolha específica dos macronutrientes após exercício, mesmo com diferenças no substrato energético utilizado durante o exercício, resultado este também reportado previamente na literatura (Alméras, lavallée, Després, Bouchard \& TREMblay, 1995).

Corroborando com nossos achados, em um estudo realizado com homens jovens, moderadamente ativos submetidos a duas sessões de exercício em esteira rolante $\left(35 \%\right.$ do $\mathrm{VO}_{2 \max }$ e $75 \%$ do $\left.\mathrm{VO}_{2 \max }\right)$ e GE de $450 \mathrm{kcal}$, não foi observado nenhuma modificação na IA total e nem na IA específica de macronutrientes (IMBEAULT et al., 1997).

\title{
Conclusão
}

De acordo com os resultados apresentados no presente estudo, podemos concluir que a ingestão alimentar pós-exercício (valor energético total e ingestão específica de macronutrientes) não se mostrou dependente da intensidade do esforço em curto prazo em indivíduos adultos jovens.

\begin{abstract}
Acute effects of exercise intensity on pos-exercise food intake in young men

The aim of this study was to investigate the acute effects of different aerobic exercise intensities (40 and $80 \%$ do $\mathrm{VO}_{2 \text { peak }}$ ) on post-exercise food intake. The sample was composed of eighteen healthy, physically active young men, of normal weight $\left(22.20 \pm 1.72 \mathrm{~kg} / \mathrm{m}^{2}\right)$. Subjects were randomly submitted to three experimental conditions: control (no exercise); low-intensity exercise (LIE $-40 \%$ of $\mathrm{VO}_{2 \text { peak }}$ ) and high-intensity exercise $\left(\mathrm{HIE}-80 \%\right.$ of $\mathrm{VO}_{2 \text { peak }}$. Exercise trials were iso-caloric $(350 \mathrm{Kcal})$. After 120 minutes of passive recovery, the volunteers had free access to a variety food buffet, and food intake was determined thru food weight. Data was analyzed by the software Nutwin 6.0 (UNIFESP, 2002) to estimate total energy intake (kcal) and consumption of the macronutrients (carbohydrates, proteins and lipids). The results suggest that food intake after exercise showed no significant differences between the experimental conditions. In conclusion, food intake after exercise is not dependent on exercise intensity in healthy young men.
\end{abstract}

UNITERMS: Energy intake; Energy metabolism; Exercise aerobic.

\section{Referências}

ALMÉRAS, N.; LAVALLÉE, N.; DESPRÉS, J.; BOUCHARD, C.; TREMBLAY, A. Exercise and energy intake: effect of substrate oxidation. Physiology \& Behavior, New York, v.57, n.5, p.995-1000, 1995.

ANÇÃO, M.S.; CUPPARI, L.; DRAIBE, A.S.; SIGULEM, D. Programa de apoio à nutrição NutWin: versão 6.0. São Paulo: Departamento de Informática em Saúde, SPDM, UNIFESP/EPM, 2002.

BILSKI, J.; TELEGŁÓW, A.; ZAHRADNIK-BILSKA, J.; DEMBINSKI, A.; WARZECHA, Z. Effects of exercise on appetite and food intake regulation. Medicina Sportiva, Krakow, v.13, n.2, p.82-94, 2009.

BLOOM, S. Hormonal regulation of appetite. Obesity Reviews, Oxford, v.8, n.1, p.63-5, 2007.

BLUNDELL, J.E.; KING, N.A. Physical activity and regulation of food intake: current evidence. Medicine \& Science in Sports \& Exercise, Madison, v.31, n.11, p.573-83, 1999. 
BLUNDELL, J.E.; STUBBS, R.J.; HUGHES, D.A.; WHYBROW, S.; KING, N.A. Cross talk between physical activity and appetite control: does physical activity stimulate appetite? Proceedings of The Nutrition Society, London, v.62, p.651-61, 2003. BROOM, D.; BATTERHAM, R.L.; KING, J.A.; STENSEL, D.J. Influence of resistance and aerobic exercise on hunger, circulating levels of acylated ghrelin, and peptide YY in healthy males. American Journal of Physiology: Regulatory, Integrative and Comparative Physiology, Bethesda, v.96, p.29-35, 2009.

BROOM, D.R.; STENSEL, D.J. BISHOP, N.C.; BURNS, S.F.; MIASHYTA, M. Exercise-induced suppression of acylated ghrelin in humans. Journal of Applied Physiology, Bethesda, v.102, p.2165-71, 2007.

DRAPEAU, V.; KING, N.; HETHERINGTON, M.; DOUCET, E.; BLUNDELL, J.; TREMBLAY, A. Appetite sensations and satiety quotient: predictors of energy intake and wight loss. Appetite, London, v.48, p.159-66, 2007.

CHENG, M.H.; BUSHNELL, D.; CANNON, D.T.; KERN, M. Appetite regulation via exercise prior or subsequent to high-fat meal consumption. Appetite, London, v.52, p.193-8, 2009

ERDMANN, J.; TAHBAZ, R.; LIPPL, F.; WAGENPFEIL, S.; SCHUSDZIARRA, V. Plasma ghrelin levels during exercise: effects of intensity and duration. Regulatory Peptides, Amsterdam, v.143, n.1-3, p.127-35, 2007.

GEORGE, V.A.; MORGANSTEIN, A. Effect of moderate intensity on acute energy intake in normal and overweight females. Appetite, London, v.40, n.1, p.43-6, 2003.

HAGOBIAN, T.A.; SHAROFF, C.G.; STEPHENS, B.R.; WADE, G.N.; SILVA, J.E.; CHIPKIN, S.R.; BRAUN, B. Effects of exercise on energy-regulating hormones and appetite in men and women. American Journal of Physiology: Regulatory, Integrative and Comparative Physiology, Bethesda, v.296, p.R233-42, 2009.

IMBEAULT, P.; SAINT-PIERRE, S.; ALMERAS, N.A.; TREMBLAY, A. Acute effects of exercise on energy intake and feeding behaviour. British Journal of Nutrition, Wallingford, v.77, p.511-21, 1997.

JACKSON, A.S.; POLLOCK, M.L. Practical assessment of body composition. Physiology and Sports Medicine, v.13, p.76-82, 1984. KING, N.A.; BLUNDELL, J.E. High-fat foods overcome the energy expenditure induced by high-intensity cycling or running. European Journal of Clinical Nutrition, London, v.49, n.2, p.114-23, 1995.

KING, N.A.; BURLEY, V.J.; BLUNDELL, J.E. Exercise-induced suppression of appetite: effects on food intake and implications for energy balance. European Journal Clinical Nutrition, London, v.48, p.715-24, 1994.

KING, N.A.; LLUCH, A.; STUBBS, R.J.; BLUNDELL, J.E. High dose exercise does not increase hunger or energy intake in free living males. European Journal of Clinical Nutrition, London, v.51, n.7, p.478-83, 1997.

KRAUSE, M.V.; MAHAN, L.K. Alimentos, nutriçáo e dietoterapia: um livro texto do cuidado nutricional. 2. ed. São Paulo: Rocca, 1991. LINS, T.A.; NEVES, P.R.S.; COSTA, M.C.C.; PRADO, W.L. Efeitos de diferentes intensidades de exercício sobre o gasto energético e a sensação de fome em jovens. Revista Brasileira de Cineantropometria e Desempenho Humano, Florianópolis, v.12, n.5, p.350-66, 2010.

LLUCH, A.; KING, N.A.; BLUNDELL, J.E. Exercise in dietary restrained women: no effect on energy intake but change in hedonic ratings. European Journal of Clinical Nutrition, London, v.52, p.300-7, 1998.

LUCKASKI, H.C.; BOLONCHUK, W.W.; HALL, C.B.; SIDERS, W.A. Validation of tetrapolar bioelectrical impedance method to assess human body composition. Journal of Applied Physiology, Bethesda, v.60, n.4, p.1327-32, 1986.

MARAKI, M.; TSOFLIOU, F.; PITSILADIS, Y.P.; MALKOVA, D.; MUTRIE, N.; HIGGINS, S. Acute effects of a single exercise class on appetite, energy intake and mood. Is there a time of day effect? Appetite, London, v.45, n.3, p.272-8, 2005. MARTINS, C.; MORGAN, L.M.; BLOOM, S.R.; ROBERTSON, M.D. Effects of exercise on gut peptides, energy intake and appetite. Journal of Endocrinology, Bristol, v.193, n.2, p.251-8, 2007.

McCONNELL, T.R. Practical Considerations in the testing of $\mathrm{VO}_{2 \max }$ in runners. Sports Medicine, Auckland, v.5, p.57-68, 1988. MOORE, M.S.; DODD, C.J.; WELSMAN, J.R.; ARMSTRONG, N. Short-term appetite and energy intake following imposed exercise in 9- to 10-year old girls. Appetite, London, v.43, n.2, p.127-34, 2004.

POMERLEAU, M.; IMBEAULT, P.; PARKER, T.; DOUCET, E. Effects of exercise intensity on food intake and appetite in women. American Journal of Clinical Nutrition, Bethesda, v.80, n.5, p.1230-6, 2004.

RANDOMIZER. Ferramenta eletrônica para aleatorização. Disponível em: <http://www.randomizer.org>. Acesso em: 1 set. 2009. STUBBS, R.J.; HUGHES, D.A.; JOHNSTONE, A.M.; HORGAN, G.W.; KING, N.; BLUNDELL, J.E. A decrease in physical activity affects appetite, energy, and nutrient balance in lean men feeding ad libitum. The American Journal Clinical Nutrition, London, v.79, n.1, p.62-9, 2004.

UNIFESP. NUTWIN: programa de apoio à nutrição. São Paulo: UNIFESP, 2002. Disponível em: <http://www.unifesp. br/dis/produtos/nutwin/index.htm>. Acesso em: 5 jan. 2010.

VERGER, P.; LANTEAUME, M.T.; LOUIS-SYLVESTRE, J. Human intake and choice of foods at intervals after exercise. Appetite, London, v.18, n.2, p.93-9, 1992. 
LINS, T.A. et al.

Free food choices after acute exercise in men. Appetite, London, v.22, n.2, p.159-64, 1994.

WESTERTERP-PLANTENGA, M.S.; VERWEGEN, C.R.; IJEDEMA, M.J.; WIJCKMANS, N.E.; SARIS, W.H. Acute effects of exercise or sauna on appetite in obese and non obese men. Physiology \& Behavior, New York, v.62, n.6, p.1345-54, 1997. WHYBROW, S.; HUGHES, D.A.; RITZ, P.; JOHNSTONE, A.M.; HORGAN, G.W.; KING, N.A.; BLUNDELL, J.E.; STUBBS, R.J. The effect of an incremental increase in exercise on appetite, eating behaviour and energy balance in lean men and women feeding ad libitum. British Journal of Nutrition, Wallingford, v.100, n.5, p.1109-15, 2008.

\section{Agradecimentos}

Os autores agradecem a FACEPE/CNPq e a Universidade de Pernambuco pelo suporte financeiro; a diretoria da Escola Superior de Educação Física (ESEF/UPE); as nutricionistas Rosa Idalina e Roberta Costi. Especialmente a todos os voluntários.

Wagner Luiz do Prado

Escola Superior de Educação Física

R. Arnóbio Marques, 310

50100-130 - Recife - PE - BRASIL

e-mail: wagner.prado@pq.cnpq.br

Recebido para publicação: 23/07/2010

1a. Revisão: 07/ 10/2010

2a. Revisão: 22/11/2010

Aceito: 22/ 11/ 2010 